\title{
Breaking the Symmetry of Momentum Conservation Using Evanescent Acoustic Fields
}

\author{
Michael Mazilu, ${ }^{1, *}$ Andriejus Demčenko, ${ }^{2, *}$ Rab Wilson, ${ }^{2}$ Julien Reboud, ${ }^{2}$ and Jonathan M. Cooper ${ }^{2}$ \\ ${ }^{1}$ SUPA, School of Physics and Astronomy, University of St Andrews, St Andrews, KY16 9SS, United Kingdom \\ ${ }^{2}$ Division of Biomedical Engineering, School of Engineering, Rankine Building, Oakfield Avenue, \\ The University of Glasgow, Glasgow, G12 8LT, United Kingdom
}

(Received 30 May 2018; published 14 December 2018)

\begin{abstract}
Although the conservation of momentum is a fundamental law in physics, its constraints are not fulfilled for wave propagation at material boundaries, where incident waves give rise to evanescent field distributions. While nonlinear susceptibility tensor terms can provide solutions in the optical regime, this framework cannot be applied directly to acoustic waves. Now, by considering a complete representation of wave interactions and scattering at boundaries, we are able to show a generic formalism of sum-frequency mixing for the whole scattering field including all evanescent waves. This general case was studied analytically and verified both numerically and experimentally for ultrasonic waves, showing that considering evanescent waves leads to an anomalous nonlinear interaction which enhances sum-frequency generation. This new interpretation not only provides a deeper understanding of the momentum conservation laws in acoustics but also promises translation of this new understanding into optics and photonics, to enhance nonlinear interactions.
\end{abstract}

DOI: 10.1103/PhysRevLett.121.244301

The conservation of momentum is a fundamental law describing wave propagation and interactions in many fields [1-4]. However, not all waves propagate and, in optics and acoustics, inhomogeneous evanescent waves [5-7] at interfaces do not have a momentum associated with them, and thus classical conservation is not directly applicable. Within the context of nonlinear optics, specific approaches have been developed to overcome this challenge, such as the use of structured surfaces to achieve momentum conservation [8,9]. These optical nonlinear effects are described by higher order tensor susceptibilities [10], while acoustics relies on Murnaghan [11] or LandauLifshitz [12] nonlinear terms, which correspond to quadratic first order differential relationships. Such nonlinear terms cannot be locally decomposed as a power series of susceptibilities (see Supplemental Material [13]). This represents a fundamental difference between optics and acoustics; i.e., the quasiequivalence between nonlinear optics and nonlinear acoustics can only be achieved when considering infinite plane or evanescent waves.

In this context, we now provide a new perspective on both finite and infinite evanescent acoustic fields, developing an analytical framework that allows the generalization of the momentum conservation law for the case when nonresonance interactions and/or evanescent acoustic

Published by the American Physical Society under the terms of the Creative Commons Attribution 4.0 International license. Further distribution of this work must maintain attribution to the author(s) and the published article's title, journal citation, and DOI. waves participate in nonlinear sum-frequency generation. In previous studies, the nonlinear interactions considered were bound by the momentum conservation law $\left(\mathbf{k}_{1}+\mathbf{k}_{2}=\mathbf{k}_{3}\right)$, restricting their application to cases of propagating plane waves $[1,4]$. In contrast, in our study, we establish a general relationship, which allows us to take into account translational symmetry breaking, akin to the relaxation of momentum conservation law. This extends our framework to a wider range of cases where momentum is not conserved in a classical sense. These cases include nonlinear interactions between homogeneous or evanescent waves and finite cross section beams, when incident waves interact at an interface or boundary [14]. We evidence this experimentally in acoustics, relating the efficiency of the resultant generated wave to the geometry of the incident waves (see Fig. 1 and Supplemental Material [13] for further numerical simulations where the nonlinear properties from Ref. [15] have been used).

We also show how such a generalized conservation law gives rise to enhanced sum-frequency signal generation above the critical angle, a phenomena analogous to the evanescent enhancement effects observed at structured optical interfaces $[8,9]$. In the context of acoustics, we show experimentally and through a new formalism that enhancement is achieved without conserving momentum. In fact, the generalized formalism developed in our study, using acoustic cases, may open new understanding in other areas of wave propagation. For example, near-field optical evanescent waves may further enhance nonlinear optical interactions at engineered metasurfaces [16].

It is well established that, when an incident wave impinges on an interface, at or above a critical angle, then 

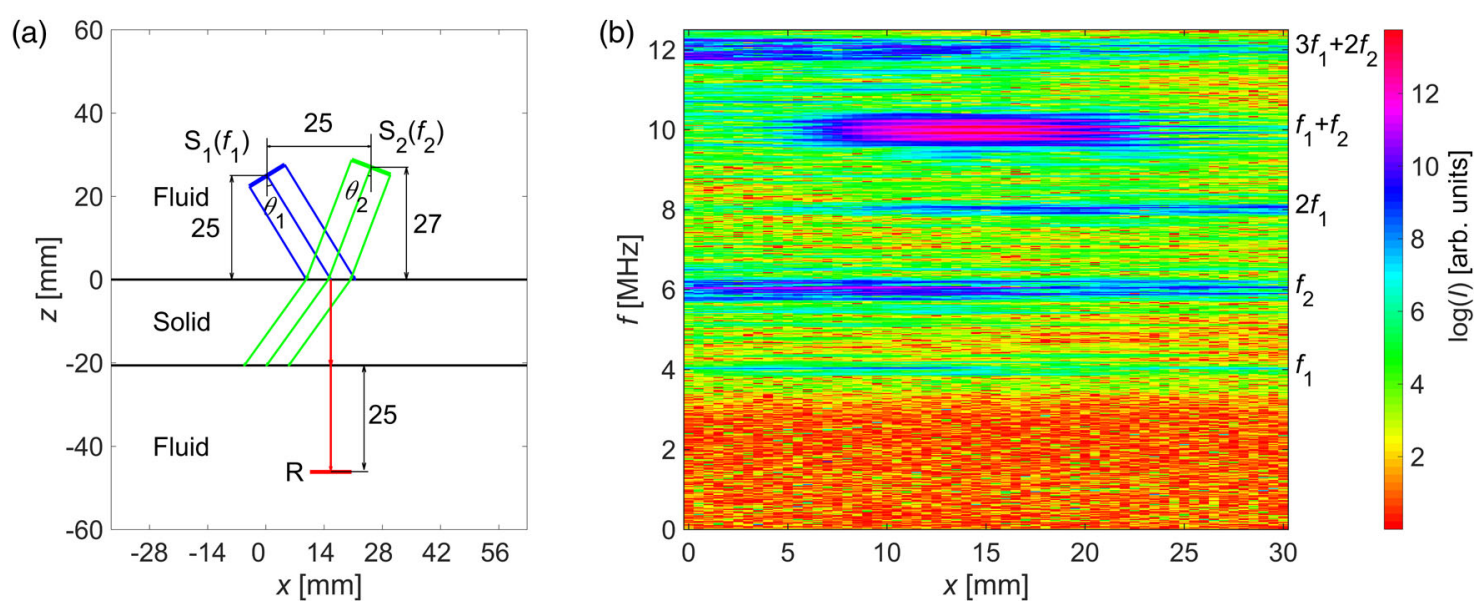

FIG. 1. Experimental configuration (a) and measured nonresonance sum-frequency generation at the water-aluminium interface (b), where $f_{1,2}$ are the frequencies of the initial waves excited by sources $S_{1}(4 \mathrm{MHz})$ and $S_{2}(6 \mathrm{MHz})$ with 10 mm active diameter, $f_{1}+f_{2}$ is the sum-frequency wave detected by the receiver $R$ with center frequency $10 \mathrm{MHz}$ and $10 \mathrm{~mm}$ active diameter, $2 f_{1}$ and $2 f_{2}$ are the second harmonics of the initial waves, $3 f_{1}$ is the third harmonic, $x$ is the detection coordinate, and $\Delta x=0.5 \mathrm{~mm} . S_{1}\left(\theta_{1}=31.5^{\circ}\right)$ excites evanescent waves (longitudinal and transversal) in the solid specimen $(d=20.6 \mathrm{~mm})$ only, while $S_{2}\left(\theta_{2}=20.8^{\circ}\right)$ excites propagating transversal waves in the solid and an evanescent longitudinal wave. At each point, the ultrasonic signal was averaged over 32 acquisitions to minimize random noise. The intensity is reported in arbitrary units (arb. units).

total reflection occurs and a nonpropagating evanescent wave on the interface is generated. In the case of acoustics, such confined waves have the potential to be used to characterize material interfaces, including high sensitivity surface sensing, subsurface profiling, interface evaluation in layered structures [17], or sensing in layered composite structures [18].

To provide a deeper understanding of these phenomena, we now present a methodology that expands and generalizes the concept of momentum conservation to take into account the finite size of the interaction region and the spatial profile of the interacting incident beams. Such interactions lead to the generation of resulting fields that break the symmetry corresponding to perfect momentum conservation [1-4]. We subsequently derive an analytical expression of the efficiency of wave generation in these relaxed conditions, using the nonlinear equation of motion [4] for an ideal isotropic solid given by

$$
\rho \frac{\partial^{2} \mathbf{v}}{\partial t^{2}}-\mu \Delta \mathbf{v}-(\lambda+\mu) \nabla(\nabla \cdot \mathbf{v})=\nabla \cdot \boldsymbol{\sigma}
$$

where $\rho$ is the volumetric density of the undeformed medium, $\mathbf{v}$ is the displacement vector in the solid, and $\lambda, \mu$ are the Lamé constants. The left-hand side of Eq. (1) corresponds to the linear wave equation and the right-hand side to the divergence of the nonlinear stress tensor (Supplemental Material [13]). This gives rise to the nonlinear bulk force, $\mathbf{F}=\nabla \cdot \boldsymbol{\sigma}$. This bulk force and the stress tensor depend on three third order elastic constants ( $A, B, C$ in Landau and Lifshitz notation [12]). Considering a plane wave having a unit polarization vector $\mathbf{u}$ and wave vector $\mathbf{k}$,

$$
\mathbf{v}=\mathbf{u} e^{-i(\omega t-\mathbf{k} \cdot \mathbf{r})},
$$

leads to the nonlinear bulk force,

$$
\begin{aligned}
\mathbf{F}= & \frac{i}{2}\{(A+2 B+2 \lambda+4 \mu)(\mathbf{k} \cdot \mathbf{k})[(\mathbf{v} \cdot \mathbf{v}) \mathbf{k}+\mathbf{2}(\mathbf{v} \cdot \mathbf{k}) \mathbf{v}] \\
& \left.+(A+6 B+4 C)(\mathbf{v} \cdot \mathbf{k})^{2} \mathbf{k}\right\},
\end{aligned}
$$

where $\omega$ is the angular frequency, $\mathbf{r}$ is the position vector, and $i=\sqrt{-1}$. We note that this equation is also valid for evanescent waves when choosing appropriate complex wave vectors.

To determine the bulk force resulting from the nonlinear interaction of two plane waves with an output wave of a combined frequency, we use the case of two plane waves generating a third wave:

$$
\mathbf{v}_{j}=\mathbf{u}_{j} e^{-i\left(\omega_{j} t-\mathbf{k}_{j} \cdot \mathbf{r}\right)},
$$

where the subscript $j$ varies from 1 to 3 . We look at the superposition of the plane waves:

$$
\mathbf{v}=\mathbf{v}_{\mathbf{1}}+\mathbf{v}_{\mathbf{2}}+\text { c.c., }
$$

where c.c. stands for the complex conjugate. To simplify, we consider the unit polarization vectors $\mathbf{u}$ to correspond to either longitudinal or shear polarizations. Using these waves and Eq. (3), we introduce the two-wave interaction stress tensor defined for any two interacting plane waves $\mathbf{v}_{\mathbf{1}}$ and $\mathbf{v}_{\mathbf{2}}$ as

$$
\sigma_{i j}^{(12)}\left(\mathbf{v}_{\mathbf{1}}, \mathbf{v}_{\mathbf{2}}\right)=\frac{1}{4}\left[\sigma_{i j}\left(\mathbf{v}_{\mathbf{1}}+\mathbf{v}_{\mathbf{2}}\right)-\sigma_{i j}\left(\mathbf{v}_{\mathbf{1}}-\mathbf{v}_{\mathbf{2}}\right)\right] .
$$


This tensor is symmetric with respect to the exchange of the two plane waves, i.e., $\sigma_{i j}^{(12)}\left(\mathbf{v}_{\mathbf{1}}, \mathbf{v}_{\mathbf{2}}\right)=\sigma_{i j}^{(12)}\left(\mathbf{v}_{\mathbf{2}}, \mathbf{v}_{\mathbf{1}}\right)$. A similar procedure can be implemented for Hermitian expressions [19] for quadratic dependencies (Supplemental Material [13]). The bulk nonlinear force

$$
\begin{aligned}
\nabla \cdot \sigma^{(12)}=\mathbf{F}^{(12)}= & e^{-i\left(\omega_{1} t-\mathbf{k}_{1} \cdot \mathbf{r}\right)} e^{-i\left(\omega_{2} t-\mathbf{k}_{2} \cdot \mathbf{r}\right)} \\
& \times \mathbf{f}^{(12)}\left(\mathbf{u}_{\mathbf{1}}, \mathbf{k}_{\mathbf{1}}, \mathbf{u}_{\mathbf{2}}, \mathbf{k}_{\mathbf{2}}\right),
\end{aligned}
$$

where $\mathbf{f}^{(12)}\left(\mathbf{u}_{\mathbf{1}}, \mathbf{k}_{\mathbf{1}}, \mathbf{u}_{\mathbf{2}}, \mathbf{k}_{\mathbf{2}}\right)$ determines the interaction strength between the two initial propagating or evanescent infinite plane waves (see Supplemental Material [13]). Importantly, $\mathbf{F}^{(12)}$ can be used for any number of waves by summing across all possible paired interactions.

In practice, we used finite cross section ultrasound beams which can be described as a superposition of plane waves. This enabled us to use an integral approach to deduce a relationship generalizing the momentum conservation law to evanescent fields for finite beams. To do this, we determined the spatially varying amplitude $a_{3}$ of the generated elastic wave at the sum frequency $\omega_{3}=\omega_{1}+\omega_{2}$ neglecting fast amplitudes variations of $a_{1}$ and $a_{2}$. This was described using the two-wave interaction term:

$$
\begin{array}{r}
\rho \frac{\partial^{2} a_{3} \mathbf{u}^{(3)}}{\partial t^{2}}-\mu \Delta\left(a_{3} \mathbf{u}^{(3)}\right)-(\lambda+\mu) \nabla\left(\nabla \cdot a_{3} \mathbf{u}^{(3)}\right) \\
=2 a_{1} a_{2} \mathbf{f}^{(12)}\left(\mathbf{u}_{\mathbf{1}}, \mathbf{k}_{\mathbf{1}}, \mathbf{u}_{\mathbf{2}}, \mathbf{k}_{\mathbf{2}}\right) e^{-i\left(\mathbf{k}_{\mathbf{1}}+\mathbf{k}_{\mathbf{2}}\right) \cdot \mathbf{r}} e^{i \mathbf{k}_{3} \cdot \mathbf{r}} \\
\quad-2 a_{1}^{*} a_{2}^{*} \mathbf{f}^{(12)}\left(\mathbf{u}_{\mathbf{1}}^{*}, \mathbf{k}_{\mathbf{1}}^{*}, \mathbf{u}_{\mathbf{2}}^{*}, \mathbf{k}_{\mathbf{2}}^{*}\right) e^{i\left(\mathbf{k}_{\mathbf{1}}^{*}+\mathbf{k}_{\mathbf{2}}^{*}\right) \cdot \mathbf{r}} e^{i \mathbf{k}_{\mathbf{3}} \cdot \mathbf{r}},
\end{array}
$$

where $a_{1}$ and $a_{2}$ are the spatially dependent amplitudes of the two interacting waves and $\mathbf{u}^{(\mathbf{3})}=\mathbf{u}_{\mathbf{3}}$ and $\mathbf{k}_{\mathbf{3}}$ is the polarization and wave vector of the generated wave at the sum frequency. The left-hand side of the Eq. (8) was further simplified by using the slowly varying envelope approximation, neglecting diffraction effects and considering the independent generation of the longitudinal and shear waves. In this case, the amplitude of the propagating generated wave was

$$
\begin{aligned}
\frac{d a_{3}}{d s}= & a_{1} a_{2} \frac{\mathbf{u}^{(3)} \cdot \mathbf{f}^{(12)}\left(\mathbf{u}_{\mathbf{1}}, \mathbf{k}_{\mathbf{1}}, \mathbf{u}_{\mathbf{2}}, \mathbf{k}_{\mathbf{2}}\right)}{2 i c_{3} \rho \omega_{3}} e^{i\left(-\mathbf{k}_{\mathbf{1}}-\mathbf{k}_{\mathbf{2}}+\mathbf{k}_{\mathbf{3}}\right) \cdot \mathbf{r}} \\
& -a_{1}^{*} a_{2}^{*} \frac{\mathbf{u}^{(3)} \cdot \mathbf{f}^{(12)}\left(\mathbf{u}_{\mathbf{1}}^{*}, \mathbf{k}_{\mathbf{1}}^{*}, \mathbf{u}_{\mathbf{2}}^{*}, \mathbf{k}_{\mathbf{2}}^{*}\right)}{2 i c_{3} \rho \omega_{3}} e^{i\left(\mathbf{k}_{\mathbf{1}}^{*}+\mathbf{k}_{2}^{*}+\mathbf{k}_{\mathbf{3}}\right) \cdot \mathbf{r}},
\end{aligned}
$$

where $s$ is the distance along the direction of propagation of the generated wave defined by the wave vector $\mathbf{k}_{\mathbf{3}}$ while $c_{3}$ is its velocity (longitudinal or shear, denoted $L$ and $S$ in the rest of the text, respectively). The superscript asterisk denotes the complex conjugate. Equation (9) defines the efficiency of generation of the sum-frequency wave for a given direction and polarization, irrespective of the nature of the interacting waves (propagating or evanescent) or the volume of the interaction region. The generated waves need not fulfill perfectly the momentum conservation conditions when considering finite interaction volumes and/or in the presence of evanescent waves. In these cases, the phase mismatch determines the resultant integrated amplitude $A_{3}$ of the generated far-field plane wave:

$$
\begin{aligned}
A_{3}= & \frac{a_{1} a_{2} \mathbf{u}_{\mathbf{3}} \cdot \mathbf{f}^{(\mathbf{1 2})}\left(\mathbf{u}_{\mathbf{1}}, \mathbf{k}_{\mathbf{1}}, \mathbf{u}_{\mathbf{2}}, k_{\mathbf{2}}\right)}{i c_{3} \rho \omega_{3}} \int_{V} e^{i\left(-\mathbf{k}_{1}-\mathbf{k}_{\mathbf{2}}+\mathbf{k}_{3}\right) \cdot \mathbf{r}} d x^{3} \\
& -\frac{a_{1}^{*} a_{2}^{*} \mathbf{u}_{\mathbf{3}} \cdot \mathbf{f}^{(\mathbf{1 2})}\left(\mathbf{u}_{\mathbf{1}}^{*}, \mathbf{k}_{\mathbf{1}}^{*}, \mathbf{u}_{\mathbf{2}}^{*}, \mathbf{k}_{\mathbf{2}}^{*}\right)}{i c_{3} \rho \omega_{3}} \int_{V} e^{i\left(\mathbf{k}_{\mathbf{1}}^{*}+\mathbf{k}_{\mathbf{2}}^{*}+\mathbf{k}_{\mathbf{3}}\right) \cdot \mathbf{r}} d x^{3},
\end{aligned}
$$

where $V$ is the interaction volume (note that $\mathbf{k}_{\mathbf{3}}$ keeps sign and $\mathbf{u}_{3}$ is not conjugated). When this volume is the whole space, then the integral evaluates to a Dirac distribution, perfect conservation relations are fulfilled, and translational symmetry is not broken. For finite volumes, this integral leads to a $k$-space (wave-vector reciprocal space) distribution that describes the coupling efficiency between the different momenta and polarizations involved. For integration, see Supplemental Material [13].

This integral does not diverge and is finite when considering the interaction between evanescent and propagating waves, although the evanescent waves break the translational symmetry and, therefore, classical momentum conservation rules $\left(\mathbf{k}_{\mathbf{1}}+\mathbf{k}_{\mathbf{2}}=\mathbf{k}_{\mathbf{3}}\right)$ do not apply.

We determined the resultant integrated amplitude $A_{3}$ for each possible combination of refracted beam and generated beam [e.g., $\left.A_{3}(L L: L), A_{3}(L S: L), \ldots\right]$, all together 8 terms. Note that these 8 terms do not describe other nonlinear processes such as second harmonic generation which can be considered using a similar approach. These analytical expressions are useful to show the fundamental origin of the sum-frequency generation involving evanescent waves, demonstrating that the phase structure of the nonlinear interaction needs to match the finite size of the overlap region and the generated mode to enable efficient generation of the resulting waves. However, the expression involves a number of approximations that are not necessarily fulfilled in realistic systems, such as multiple reflection and diffraction. To account for these effects we used finite element methods (COMSOL MULTIPHYSICS ${ }^{\circledR}$ version 5.2a) to model a realistic generation of the sum-frequency signal and its propagation.

Here, we considered a single interface between a fluid and a solid semispace [see Fig. 2(a)]. We carried out the experiments with well-known materials, such as water and aluminium. In this case, two acoustic beams were generated in the water and refracted on a planar interface. This refraction effect was calculated analytically while neglecting diffraction. In the aluminium half-space, there are four refracted waves corresponding to a longitudinal and shear wave from each of the two incident beams. As the 

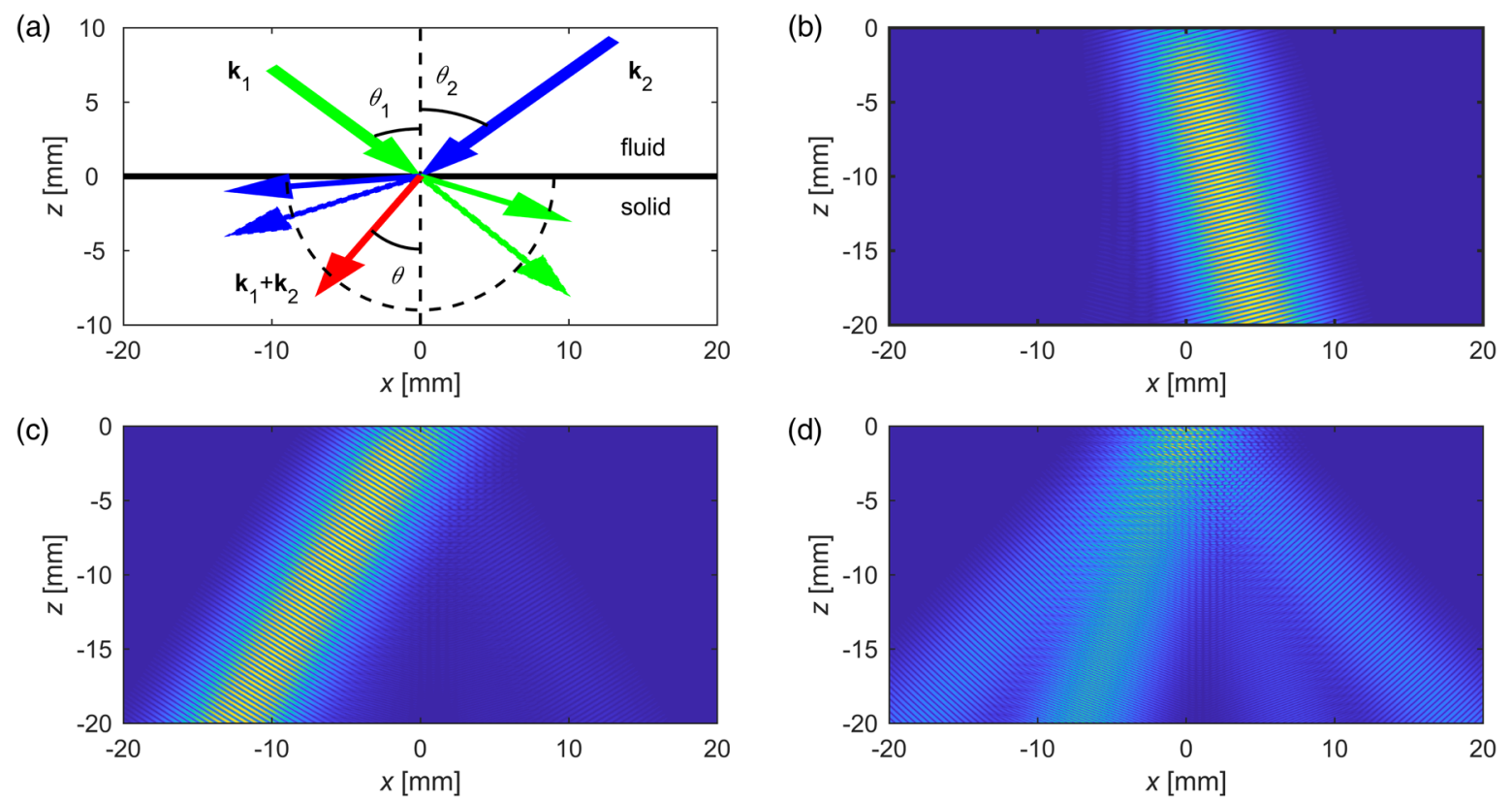

FIG. 2. (a) Illustration of two incident elastic beams (blue and green) interacting after refraction at the fluid-solid interface to generate the sum-frequency beam (red). (b)-(d) Total displacement distribution at the sum frequency for three incidence angles of the second beam $\left(-8.5^{\circ},-26.8^{\circ}\right.$, and $\left.-30.5^{\circ}\right)$. All angles are measured with respect to the normal and positive in the anticlockwise direction.

incidence angle was varied, these refracted beams were either propagating or evanescent. We note that the shear wave velocity in aluminium is larger than the speed of sound in water and therefore any generated shear wave refracts as a longitudinal wave in water.

In the simulations, we chose $\theta_{1}=21.5^{\circ}$ and $\theta_{2}$ varies from $0^{\circ}$ to $-60^{\circ}$, while $\theta$ defines the angle of the generated sum frequency [see Fig. 2(a)]. Figures 2(b) and 2(c) depict the case where the interacting waves are propagating and the generated waves fulfill the momentum conservation relationship $\left[S\left(\omega_{1}\right)+L\left(\omega_{2}\right) \rightarrow L\left(\omega_{1}+\omega_{2}\right)\right.$ and $S\left(\omega_{1}\right)+$ $S\left(\omega_{2}\right) \rightarrow L\left(\omega_{1}+\omega_{2}\right)$ abbreviated as $S L: L$ and $\left.S S: L\right]$. Strict momentum conservation was not possible when the incident beams were above the critical angle leading to evanescent waves at the interface. Figure 2(d) shows the case when $\theta_{2}$ was above the critical angle and the interaction occurred mainly between shear propagating $S\left(\omega_{1}\right)$ and longitudinal and shear evanescent $E\left(\omega_{2}\right)$, beams generating longitudinal and shear waves at the sum frequency.

One way to visualize the angular dependence of the sum-frequency generation is to evaluate the tangential and radial displacements on a semicircle [black dashed in Fig. 2(a)]. The intensity of these displacements is shown in Fig. 3 for the two possible polarizations of the generated beam. The expected interactions $S L: L$ [Fig. 3(a)] and $S S: L$ [Fig. 3(b)] for propagating beams are observed.

When one wave is evanescent Fig. 3(c), the interactions $S E: S$ and $S E: L$ take place simultaneously, generating propagating waves in two different directions due to the different propagation phase velocities resulting from each interaction. Indeed, constructive interference occurs only in the direction that fulfills the momentum conservation
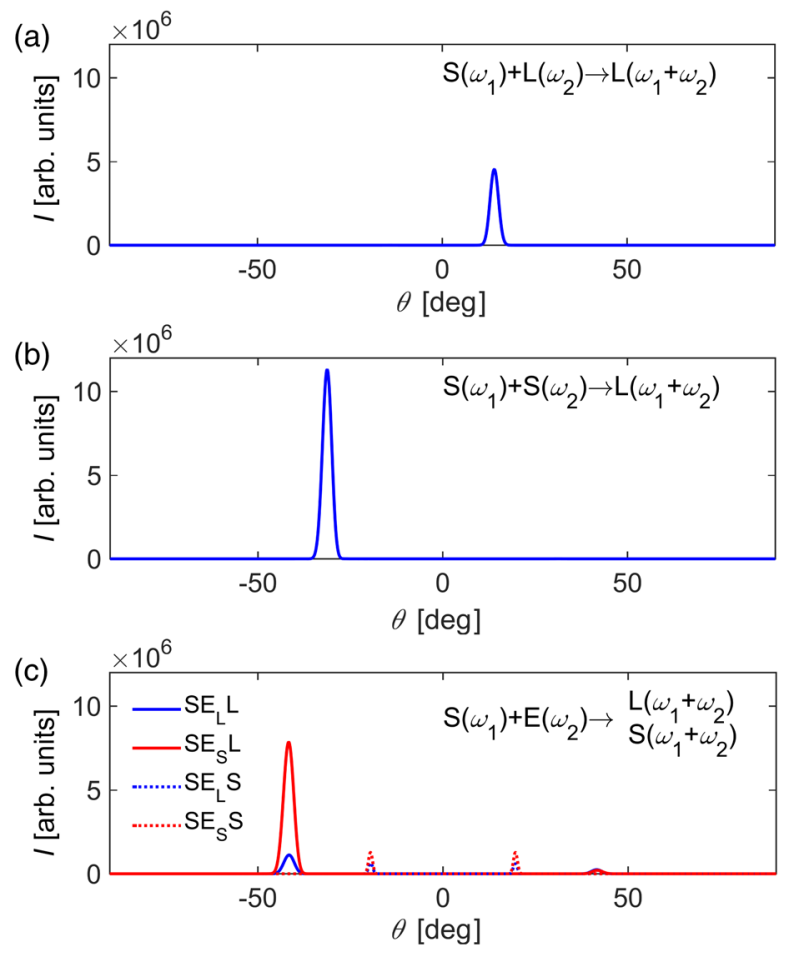

FIG. 3. Angular intensity dependence at the sum frequency for three incidence angles of the second beam (a) $-8.5^{\circ}$, (b) $-26.8^{\circ}$, and (c) $-30.5^{\circ}$. Intensity is reported in arbitrary units (arb. units). 

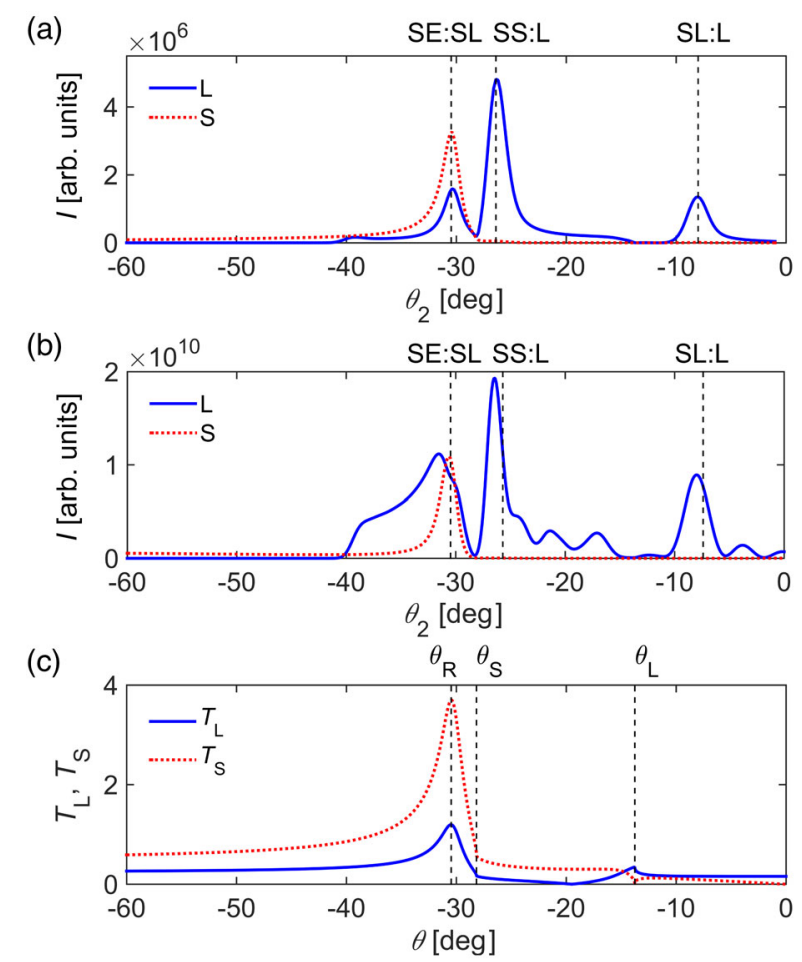

FIG. 4. Total intensities of generated waves using (a) our finite elements method and (b) our analytic model. The vertical dotted lines (a), (b) indicate the peak positions in (a). (c) Amplitude of the displacement transmission coefficients for longitudinal (blue) and shear (red) polarization plane waves. The dotted lines indicate critical angles for the three waves (longitudinal $\theta_{L}$, shear $\theta_{S}$, and Rayleigh $\theta_{R}$ ).

relationship or, in the case of evanescent waves, over volumes smaller than the coherence length. To obtain a general understanding, beyond the discrete cases of wave interactions, we calculated the generated sum-frequency signal integrated along the semicircle described by Fig. 2(a) using the numerical model, Fig. 4(a), while Fig. 4(b) shows the analytically calculated far-field distribution using Eq. (10). These integrated signals were represented as a function of the angle of incidence of the second beam $\theta_{2}$. For specific angles, phase matching conditions are reached that lead to a classic nonlinear wave interaction described by the momentum conservation relation. The resonance conditions in this case are $S L: L-8.5^{\circ}$ and $S S: L-26.8^{\circ}$. Further sum-frequency generation intensity increase was observed when one of the refracted beams was close to the critical angle, $\theta_{R}$. At this angle, the generation efficiency increased due to the surface field enhancement linked to the evanescent wave and the increase in transmission coefficients; see Fig. 4(c). These results were verified experimentally (see last section of Supplemental Material [13]).

The nonlinear mixing between a propagating bulk wave and an exponentially decaying wave couples to a bulk shear and longitudinal mode at the sum frequency. This coupling occurs near the surface, in a small volume defined by the skin depth $[20,21]$. The interaction is enhanced by the fast variation of the intensity envelope of the incident beams, leading to additional anomalous contributions from Murnaghan's nonlinear terms [right-hand side of Eq. (9)]. This anomalous contribution was observed experimentally (see last section of Supplemental Material [13]). In nonlinear optics, the description of these interactions is different as, contrary to acoustics, higher order nonlinear susceptibility terms are used. The new framework developed here complements this description in optics, and thus opens up new opportunities for study beyond acoustics.

In conclusion, we have expressed a new framework for generalized momentum conservation as a $k$-space distribution that describes the coupling efficiency between the different momenta involved. This expression collapses to a Dirac distribution in the case of infinite propagating planewave interactions. However, in contrast, finite interaction volumes and evanescent waves lead to a nonsingular distribution. Our new framework comes from a general description of nonlinear wave scattering, which consequently promises new insight into nonlinear optics.

All data created during this work are available open access from the University of Glasgow [22].

J.M.C. acknowledges EPSRC Fellowship (EP/ K027611/1) and J. M. C., J. R., A. D., and R. W. thank the ERC advanced investigator award (340117-Biophononics) for support.

*M. M. and A. D. contributed equally to this work.

[1] L. H. Taylor and F. R. Rollins, Phys. Rev. 136, A591 (1964).

[2] J. M. Rouvaen, R. Torguet, E. Bridoux, and C. Bruneel, J. Appl. Phys. 48, 934 (1977).

[3] J. B. Bertrand, H. J. Wörner, H.-C. Bandulet, É. Bisson, M. Spanner, J.-C. Kieffer, D. M. Villeneuve, and P. B. Corkum, Phys. Rev. Lett. 106, 023001 (2011).

[4] V. A. Korneev and A. Demčenko, J. Acoust. Soc. Am. 135, 591 (2014).

[5] S. Choudhary and L. B. Felsen, IEEE Trans. Antennas Propag. 21, 827 (1973).

[6] L. B. Felsen, J. Opt. Soc. Am. 66, p751 (1976).

[7] N. F. Declercq, R. Briers, J. Degrieck, and O. Leroy, IEEE Trans. Ultrason. Ferroelectr. Freq. Control 52, 776 (2005).

[8] M. Schnippering, S. Neil, S. MacKenzie, and P. Unwin, Chem. Soc. Rev. 40, 207 (2011).

[9] J. Renger, R. Quidant, N. Van Hulst, and L. Novotny, Phys. Rev. Lett. 104, 046803 (2010).

[10] R. W. Boyd, Nonlinear Optics, 3rd ed. (Academic Press, Boston, 2008).

[11] F. D. Murnaghan, Am. J. Math. 59, 235 (1937).

[12] L. D. Landau and E. M. Lifshitz, Theory of Elasticity (Pergamon Press, London, 1959).

[13] See Supplemental Material at http://link.aps.org/ supplemental/10.1103/PhysRevLett.121.244301 for methods, numerical modeling, theory and experimental results.

[14] L. K. Zarembo and V. A. Krasil'Nikov, Sov. Phys. Usp. 13, 778 (1971). 
[15] J. Asay and A. H. Guenther, J. Appl. Phys. 38, 4086 (1967).

[16] A. Krasnok, M. Tymchenko, and A. Al, Mater. Today 21, 8 (2018).

[17] E. Escobar-Ruiz, A. Ruiz, W. Hassan, D. C. Wright, I. J. Collison, P. Cawley, and P. B. Nagy, J. Nondestruct. Eval. 33, 187 (2014).

[18] A. Demčenko, V. Koissin, and V. A. Korneev, Ultrasonics 54, 684 (2014).
[19] M. Mazilu, J. Baumgartl, S. Kosmeier, and K. Dholakia, Opt. Express 19, 933 (2011).

[20] M. A. Seo, H. R. Park, S. M. Koo, D. J. Park, J. H. Kang, O. K. Suwal, S. S. Choi, P. C. M. Planken, G. S. Park, N. K. Park, Q. H. Park, and D. S. Kim, Nat. Photonics 3, 152 (2009).

[21] S. I. Rokhlin and Y. J. Wang, J. Acoust. Soc. Am. 89, 503 (1991).

[22] See http://dx.doi.org/10.5525/gla.researchdata.689. 